\title{
Sinderos
}

\section{Socialización mediática infantil: Un acercamiento teórico-conceptual}

Children's media socialization: A theoretical-conceptual approach

Autora:

Sara Carolina Carrillo David ${ }^{1}$

Recibido: 12 de mayo de 2017

Aprobado: 16 de mayo de 2018

1. Licenciada en Pedagogía Infantil de la Universidad de Antioquia. Magíster de la U. de A. y estudiante del doctorado en Educación de la U. de A. Docente de cátedra de la U. de A. y docente de Educación Infantil.

\section{Resumen}

Este trabajo pretende mostrar la relación entre socialización infantil, medios de comunicación (media) y educación, asumiendo que la socialización mediática no hace referencia a un mero proceso de adaptación. La noción de socialización mediática infantil se profundiza como un potencial investigativo que les importa a la sociología y a la pedagogía como campo conceptual. Las líneas de trabajo que se proponen al final parten de una revisión teórica sobre socialización y socialización mediática.

Palabras clave: Socialización infantil, medios de comunicación, socialización mediática 


\begin{abstract}
The purpose of this work is to show the relationship between child socialization, media (media) and education, based on the idea that media socialization does not refer to a mere process of adaptation. The notion of children's media socialization deepens as a research
\end{abstract}

potential that matters as a conceptual field to sociology and pedagogy. The lines of work proposed at the end of the article are based on a theoretical review on socialization and media socialization.

Keywords: Child socialization, media, media socialization

\section{Introducción}

El propósito de este trabajo es mostrar la relación entre socialización infantil, medios de comunicación (media) y educación. En el trabajo se sustenta la tesis según la cual la socialización mediática no es un simple proceso adaptativo y que los medias hoy en día, además de fungir como agentes socializadores, se han vuelto también parte inmanente de otras instancias (agentes, espacios) socializadoras como la familia y la escuela. Para ello se trabaja conceptualmente la noción de socialización mediática infantil y se muestra el potencial investigativo de dicho concepto para la investigación sociológica y pedagógica. En la primera parte se hace una revisión a las diferentes concepciones y planteamientos de la socialización y la socialización mediática y se propone un punto de vista anclado en la apropiación mediática. En la segunda parte se señalan las perspectivas de trabajo (a manera de programática) que se abren para un estudio de la socialización mediática y se concluye sobre el papel de la pedagogía como campo de investigación sobre dichos asuntos.

\section{Socialización y socialización mediática: aspectos conceptuales}

La socialización es un concepto básico dentro de las ciencias sociales y humanas (Brigido 2006; Dubet y Martuccelli, 1996). Bajo el concepto de socialización se conciben todos los procesos a partir de los cuales los individuos devienen o se vuelven sociales. Como lo dicen Arne Biederbacher y Peter Zimmermann (2010, p. 12), por socialización se puede entender el "proceso de desarrollo de la personalidad en dependencia del entorno social (mediado histórica y socialmente)". La socialización, desde una perspectiva social y pedagógica, tiene que ver con cuestionamientos como los siguientes: ¿cómo y en qué medida el infante deviene en un sujeto social, capaz de acción y autónomo a partir de su nacimiento? Es decir, ¿cómo le llega el mundo social, histórico, cultural - al individuo? Con base en estos cuestionamientos se han establecido, dentro de la sociología y la pedagogía — pero también la psicología-, diferentes planteamientos entre los que cabe destacar tres orientaciones básicas: el planteamiento funcional, el planteamiento sociocultural y el planteamiento ecológicosocial (Süss, 2004, p. 25). A continuación vamos a referirnos sucintamente a dos de ellos para mostrar su importancia en lo que tiene que ver también con una propuesta de socialización mediática. 


\section{Planteamiento funcional (La socialización como adaptación)}

La respuesta clásica a la pregunta por la socialización la desarrolla por primera vez Emile Durkheim en el marco histórico del proceso de modernización de la sociedad industrial. Para Durkheim, la integración social sólo es posible "cuando todos los miembros de la sociedad interiorizan las normas y los mecanismos de coacción, cuando la sociedad hasta cierto punto los penetra o entra en ellos y organiza a partir de allí su personalidad" (Hurrelmann, 2006, p. 12). La socialización es, sobre todo desde este punto de vista, una adaptación de los individuos a las representaciones sociales y un encadenamiento e integración a las estructuras existentes. A través de ello la socialización es vista, sobre todo, como una influencia sobre el individuo y una representación de los valores y normas sociales.

A teóricos de la socialización, como Durkheim (1976) y, posteriormente, Parsons (1956, 1999), los caracteriza una posición denominada conservadora, pues sus preocupaciones se orientan más hacia el aseguramiento de la objetividad de los hechos sociales — hacia el mantenimiento de las instituciones sociales- por encima de los individuos - y de sus intereses y motivaciones-. Para estas perspectivas sociológicas el individuo se presenta más como una amenaza al orden establecido y, por tanto, las instituciones se tienen como instancias que contribuyen a mitigar tales potenciales de amenaza mediante procesos de socialización disciplinada y socialización metódica (educación) que permitan una adecuada integración social.

\section{Planteamiento ecológico-social (la socialización como creación social de redes)}

Con las consideraciones acerca de los procesos de individualización en las sociedades contemporáneas y con los planteamientos de la "nueva sociología de la infancia" con respecto a los niños y niñas como agentes, las posiciones funcionales clásicas acerca de la socialización, como las de Durkheim y Parsons, han experimentado cada vez más críticas. Se ha llegado a la idea de que el sujeto, particularmente el infante, en las teorías de la socialización ha de ser visto como un sujeto actuante y activo. Teorías actuales de la socialización, como las de Arne Niederbacher y Peter Zimmermann (2011) y las de Klaus Hurrelmann (2006), por ejemplo, parten del presupuesto de que la socialización "sería un proceso de elaboración de toda la vida respecto a las exigencias externas e internas referidas al desarrollo de la personalidad" (Hurrelmann, 2006, p. 20), lo cual estaría:

(1) en contra de una concepción biologicista del desarrollo humano y en contra de una determinación por las 'disposiciones' y la 'maduración'. Aunque haya que considerar, igualmente, que el ser humano es un ser biológico; (2) en contra de la concepción idealista del sujeto. (3) No hay 'individuo libre' que se pueda escapar a los influjos sociales y a las explicaciones científicas. Se tiene que considerar, no obstante, que el ser humano no se encuentra determinado de un modo mecánico por el entorno (del que procede $-\mathrm{y}$ en el que crece-), sino 
que es un ser que actúa intencionalmente y de modo reflexivo; (4) en contra de una reducción pedagógica del desarrollo infantil, que solo considera la relación educador y pupilo. Pero, igualmente, la interacción entre infantes y adultos (maestros, padres, etc.) es un elemento fundamental y muy importante en el proceso de socialización. (Niederbacher y Zimmermann, 2011, p. 13)

A partir de este punto de vista vamos a entrar en un segundo planteamiento que sostiene que la socialización tiene lugar mediante la creación de redes sociales en un proceso complejo de intercambio entre ser humano y entorno. Este planteamiento parte del presupuesto según el cual los seres humanos se mueven en diferentes sistemas - entornos - sociales. Y para moverse dentro de esos sistemas es necesaria una competencia para comunicarse y actuar que hace posible la interacción social.

A partir de estos presupuestos básicos Urie Bronfenbrenner, uno de los fundadores de este planteamiento ecológico-social, desarrolla un modelo orientado desde una teoría de sistemas acerca de las diferentes zonas ecológico-sociales, las cuales remiten respectivamente a ciertas regularidades propias en relación con la comunicación y la interacción. A partir de acá Bronfenbrenner, en su libro La ecología del desarrollo humano. Experimentos en entornos naturales y diseñados, publicado por primera vez en 1979, divide los sistemas en: (1) microsistemas o entornos inmediatos (por ejemplo, los padres, los amigos cercanos, abuelos y colegas de trabajo), (2) mesosistemas (por ejemplo, la formación o el establecimiento de relaciones entre los microsistemas), (3) exosistemas (son sistemas en los que el sujeto no está, pero lo influyen o tienen influencia sobre él. Por ejemplo, los medios masivos), y (4) macrosistemas o patrones generalizados (sistemas más comprensivos como la sociedad, el Estado, la ideología).

En lo que respecta a la relevancia o importancia de esas zonas, Bronfenbrenner (1987) resalta que éstas cambian con el transcurso de la biografía; por ello, acá se incluye la dimensión temporal que el autor denomina cronosistema. Dentro del cronosistema se organizan las transformaciones y pasos biográficos de relevancia y definitivos en la vida de los individuos. Desde el punto de vista temporal y espacial el mesosistema se extiende toda vez que siempre entran en relación entre sí nuevos microsistemas, lo cual tiene que ver con los procesos de socialización. De un modo resumido, el proyecto ecológico-social tiene como propósito: "proporcionar un esquema conceptual unificado pero muy diferenciado, para describir e interrelacionar estructuras y procesos, tanto en el ambiente inmediato como en el más remoto, que va dando forma al curso del desarrollo humano durante toda la vida [...]" (Bronfenbrenner, 1987, pp. 30, 32).

De una manera análoga al modelo de Bronfenbrenner, Dieter Baacke ha tratado de construir una propuesta que comprende el mundo de la vida (de los infantes) en cuatro zonas (Baacke, 1991, p. 96; 1998, p. 111). Si bien tales zonas pueden ser delimitadas por cuestiones analíticas, se han de entender como interdependientes. La idea de interdependencia remite a que el sujeto (el niño o la niña) va descubriendo más su mundo vital o su entorno y que, en ese sentido, se desarrolla de adentro (los 
padres) hacia afuera (los pares y las instituciones). Ello conduce a una diferenciación de esas zonas.

Baacke denomina a la primera zona de su modelo el centro ecológico, que es representado por la familia (es decir, por el hogar). Se trata de aquel entorno en el cual se encuentran las personas de referencia más importantes. Esta primera zona, el centro ecológico, es el espacio circundante y cercano más inmediato y cotidiano; aquel en el que nacemos. Desde el punto de vista espacial sigue, en la segunda zona, el vecindario, el entorno barrial o los alrededores de la vivienda que el infante vincula con el primero en una relación de adentro y afuera. Baacke denomina esta zona el espacio ecológico próximo o cercano y se puede entender como el entorno inespecífico de la zona uno. En ella se llevan a cabo las primeras relaciones externas más allá del entorno inmediato. El niño se encuentra aquí con sus compañeros de juego, con sus amistades, etc. Los alrededores de la casa (el barrio o el vecindario) se vuelven para los infantes en una rivera más o menos estructurada con diferentes puntos de encuentro. Así, mientras más puntos de encuentro estén a disposición más cerca estará ese alrededor de volverse en un óptimo ecológico. La tercera zona es menos vinculante, es decir, se puede definir mediante relaciones funcionales específicas, y tiene que ver con las unidades ecológicas que representan lugares determinados. Ejemplo de ello son el jardín infantil, la escuela, la comunidad eclesiástica, los clubes deportivos, las tiendas, los lugares comerciales, entre otros. La determinación de estos espacios tiene lugar mediante la funcionalidad que se les atribuye y que permite sancionar los comportamientos y actividades. Por tanto, en estos espacios los niños y las niñas tienen que actuar en concordancia con ciertos roles. En la cuarta zona, la zona de la periferia ecológica, se encuentran espacios más allá de la cotidianidad normal como los lugares de vacaciones, los lugares de descanso, los lugares para el tiempo libre o los "lugares prohibidos". Esta cuarta zona debe ser considerada como la periferia ecológica de los contactos esporádicos u ocasionales y como un entorno de excepciones. Mientras más variada y rica la periferia ecológica, más abiertos y experimentados se vuelven los infantes. En ese sentido, una periferia ecológica rica y variada no sólo amplía el radio del ámbito de acción de los infantes, sino que, gracias a ella, estos adquieren más posibilidades de evasión y alternativas respecto a su entorno inmediato. Mientras más libertad de movimiento y de oportunidades para actuar y comunicarse procuradas por estas diferentes zonas ecológicas, más reforzados y exigentes se vuelven los procesos de socialización y más complejo el desarrollo de la personalidad.

El planteamiento ecológico-social resulta particularmente interesante para la pedagogía y para la praxis educativa, en la medida en que con él se consideran las dificultades en el proceso de socialización (ligadas a la formación, el aprendizaje y el desarrollo) como cuestiones que no se pueden tratar aisladamente y que, por el contrario, exigen prestarles atención a los influjos familiares y del entorno. Así, el proceso de socialización ha de tenerse como 
un acontecimiento activo que consiste en la elaboración productiva de realidades internas y externas. [...] Los seres humanos no son sólo socializados, sino que se socializan a sí mismos. Ellos escogen los entornos que les agradan (a partir de sus propios intereses) y establecen resistencias con los entornos que no les corresponden. La investigación sobre la socialización se tiene que ocupar de la apropiación de aspectos simbólicos y materiales del mundo y considerar las formas de control, de direccionamiento (educación) y de resistencia y de emancipación. (Süss, 2004, p. 32)

Con la perspectiva ecológico-social se resalta que los infantes no simplemente se adaptan a su entorno, sino que lo confrontan de una manera activa y le dan forma. En ese sentido, no se trata de la adaptación del individuo al entorno sino de un ajuste entre el individuo y su entorno. La socialización es por ello un proceso de toda la vida que tiene que ver con la estructuración y orientación en el mundo y en la que la personalidad adquiere un papel central. En la medida en que la socialización en los espacios sociales se puede entender como un proceso de formación (subjetivación) y de aprendizaje con un entorno concreto, entonces la acción social (interacciones sociales), desde el punto de vista de la ecología social, se puede concebir como un proceso activo e interactivo de confrontación con el entorno.

Un planteamiento ecológico-social con respecto a los medios resulta particularmente aportante para pensar la relación entre infancia, medios y socialización, y se diferencia fuertemente con respecto a otras posturas que plantean criterios cuasi objetivos o mono-causales acerca del efecto de los medios sobre los individuos. En este planteamiento los comportamientos, las acciones y las interacciones no se dejan explicar simplemente de una manera causal como simples influjos sociales o ambientales sobre los individuos y, en ese sentido, va más allá de la socialización como simple integración social, a una mirada a la socialización como reinterpretación y resignificación productiva del mundo y como apropiación significativa de los entornos sociales. Más bien la situación, el contexto socioespacial, filtra esos influjos en la interacción de las personas en su conjunto, lo que permite entender a los individuos como partícipes de su propio proceso de socialización. De allí que el uso de los medios sea siempre contextualizado, situado y orientado de una manera cultural e incluso emocional. Por eso, en la investigación, esos contextos tienen que ser reconstruidos si se quiere hacer afirmaciones o enunciados sobre la socialización mediática de la infancia.

\section{Socialización mediática: cuestionamientos orientadores y perspectivas}

Cuando se mira desde el punto de vista social y pedagógico el papel de los medios en relación con los seres humanos, a menudo esto se comprende con el concepto de socialización mediática (Süss, Lampert y Wijnen, 2010, pp. 29 y ss.). Sin embargo, detrás de dicha expresión no hay un concepto unitario, sino un espectro de teorías diferentes, unas surgidas en el marco de la pedagogía y otras, en el marco de la sociología, de la comunicación, de las teorías de la recepción o de la psicología comunicacional. 
Como venimos viendo, el planteamiento al que nos adherimos está relacionado con una mirada ecológico-social con un énfasis microsociológico. Se trata de una perspectiva que se puede anclar al paradigma hermenéutico y a las investigaciones que tienen que ver con el redescubrimiento de las teorías de lo cotidiano y del mundo de la vida dentro de la tradición hermenéutica (Kleemann, Krähnke y Matuschek, 2013). Esto está en relación también con los usos de los métodos cualitativos en las ciencias sociales y humanas. Lo que caracteriza estos pensamientos es la preocupación por fenómenos microsociales —no necesariamente desatendiendo o despreocupándose de los aspectos macroestructurales-. En ese sentido, aquí la realidad social es explicada no sólo a partir de investigaciones referidas a las estructuras sociales globales, sino mediante la explicación del transcurrir de la vida cotidiana y de las reglas e interacciones que organizan la interacción y la acción cotidianas. En esa lógica se puede definir y tratar la socialización mediática como un asunto de investigación desde dos perspectivas programáticas:

- Una en la que se observa el influjo sobre los sujetos por parte de los medios. Esto gira en torno a preguntas como: ¿qué hacen los medios con los seres humanos (efectos de los medios)?, ¿cómo cambian los medios los procesos de socialización en general y son estos cambios promotores del desarrollo de la personalidad o más bien amenazas o peligros (efectos de los medios)?

- Otra en la que se plantea una elección activa de los medios y una elaboración de los contenidos por parte de los sujetos. Esto gira en torno a preguntas como las siguientes: ¿qué hacen los seres humanos con los medios (selección y uso de los medios)?, ¿cómo aprenden los seres humanos a tratar o arreglárselas con los medios y qué formas de tratos se pueden diferenciar (competencias mediáticas)?

A partir de este punto se pueden identificar otras perspectivas que tienen que ver con la utilización de los medios:

- Lo que tiene que ver con la socialización (elección de los medios, si se trata de decisiones autónomas o prescritas),

- El trato con el mundo del consumo (los medios como mercancías, todo el problema de los consumidores y todo lo que tiene que ver con los costos),

- La participación política (mantenimiento de ciertos valores, intereses, contenidos políticos, el comportamiento en el tiempo libre),

- La búsqueda de gratificaciones (necesidades, influjos, motivos, comunicación, público),

- El contexto de generación de los medios (lugar de los medios, valores, formas de pensar, comportamientos, contenidos de los medios, riesgos, utilidades, metas, intereses de búsqueda).

Siguiendo a Kübler (2009, pp. 18 y ss.) planteamos acá que las investigaciones sobre la socialización mediática, más que estar integradas a una "gran" teoría unificada de la socialización mediática, representan esfuerzos desde diferentes perspectivas disciplinarias, enfoques y metodologías, por lo que cobra importancia con ello una preocupación más por problemas que por el desarrollo de una teoría unificada. 


\section{Socialización y medios (o apropiación mediática)}

Una manera de poner en consonancia "medios" y "socialización” viene dada por el concepto de apropiación de los medios, cuyos principales exponentes son Bernd Schorb y Helga Theunert (1995). Si bien, a menudo, se utiliza para ello el concepto de internalización, en el sentido de normas que se viven como propias, es decir, que se internalizan, el concepto de apropiación de medios o mediática enfatiza en la capacidad activa del sujeto y conceptúa la acción mediática como una parte del actuar social. La socialización mediática se puede entender desde esta perspectiva como una relación bidireccional entre el sujeto y los medios. Mientras los individuos se apropian de maneras diferentes y de modo intersubjetivo de un medio, también están expuestos, al mismo tiempo, al influjo de esos medios. Por ejemplo, los medios determinan los procesos de apropiación en lo referente al contenido, en la medida en que prescriben unas imágenes y no otras e influyen sobre las formas y maneras de actuar que no están relacionadas con los medios; estas imágenes prescritas tienen que ver con muchos ámbitos de la vida cotidiana. Esto ha suscitado en muchos marcos de discusión, en relación con la socialización mediática, los debates sobre todo respecto a los efectos negativos de los medios, es decir, los medios muestran imágenes de violencia que los niños y las niñas repiten en sus situaciones cotidianas, o siguen modelos de comportamiento presentados a través de ellos que marcan los comportamientos en la cotidianidad. Sin embargo, también se puede decir que los medios tienen un efecto positivo en los niños y las niñas. Los medios no despliegan sólo sus efectos negativos, sino que entran a cumplir un papel importante en relación con otros factores: por ejemplo, pueden tener un efecto de refuerzo sobre ciertas disposiciones y, así como la familia y el círculo de amigos, se constituyen en un factor socializador autónomo. En ese sentido, los medios no son sólo intermediarios, portadores o vehículos dentro de los procesos de socialización, sino que son además objeto de los procesos de socialización.

Hablando desde una perspectiva ecológico-social, los medios, en el proceso de socialización, también constituyen un espacio que configura diferentes situaciones. En situaciones específicas los medios - ligados a los artefactos- hacen que lo que esté sucediendo se tenga que organizar de una manera tal que los que actúan o interactúan allí lo tienen que hacer de una forma adecuada y en concordancia con la situación. Un ejemplo de ello es cuando se ve la televisión: los sujetos aprenden a reaccionar de maneras específicas cuando están viendo noticias o cuando están viendo una película de ficción.

Científicos sociales y pedagogos (Petit, 2009; Moser, 2010; Süss, Lampert y Wijnen, 2010; Graviz, 2010; Racioppe, 2008) ven los media como una instancia socializadora de la infancia junto con la familia, los pares, la escuela y otros agentes. Los media y sus tecnologías permiten la construcción de los mundos sociales, marcan e influyen sobre el comportamiento de los infantes y, por su multiplicidad de funciones, hoy en día son acompañantes permanentes de niños y niñas. La socialización infantil se ve cada vez más influenciada por los medios y las tecnologías de la comunicación. 
En ese sentido, los medios y las tecnologías fungen como factores mediadores de la socialización, pero también como instrumentos (artefactos) en el proceso de socialización infantil. Se trata de procesos de socialización que cobran cada vez más una relevancia pedagógica.

Muchos estudios muestran que la cotidianidad infantil es cada vez más atravesada y marcada por los medios electrónicos (Buckingham, 2002). Se conoce además que las configuraciones mediales de nuestro entorno tienen influjo sobre nuestras maneras de pensar y de actuar. Los medios electrónicos permiten que los sujetos orienten su vida diaria (ello va desde utilizar el celular como despertador hasta establecer relaciones amorosas por medio del internet), pero también son un apoyo en el aprendizaje y procesamiento de la información (función cognitiva y epistemológica), igualmente ayudan a que los individuos se distraigan, se relajen, se diviertan, se tematicen a sí mismos y reflexionen sobre sí. Es decir, cumplen con una función afectiva, por ejemplo, en el sentido de que posibilitan que uno se "pierda" en el mundo de la fantasía y puede así vivir y tener sentimientos y vivencias intensos. Particularmente la televisión, cumple con ciertas funciones psicosociales y del desarrollo de ciertas edades. Para los infantes son factores importantes la fantasía, los controles — de los impulsos - y el progresivo descubrimiento del mundo real. Junto con la distensión y el relax que los media promueven se ejercitan, de manera lúdica, ciertas reacciones en un mundo virtual donde pueden entrar en escena y ser manejadas - o no- en situaciones reales posteriores.

Los medios también contribuyen con la formación y el establecimiento de valores (función integrativa), así como con el intercambio de experiencias e ideas (función interactiva). En ello cabe destacar su papel en: interacción social: se puede interactuar con ciertas personas si se tiene internet o si tienen un artefacto igual al que no todos pueden acceder; integración: además de servir para hacer llamadas, un celular puede ser un requisito para el ingreso a cierto grupo y fungir como criterio de adscripción e identificación con un colectivo; distinción y estatus social: una determinada marca de computador o de celular puede ser una forma de - reafirmar - distinción y estatus social: "los que tienen esto son esto"; modo de pertenencia: tener y usar un producto tecnológico puede ser un modo de pertenencia a cierto grupo, a cierta filosofía, a cierta forma o estilo de vida. Pero también se puede ver en ellos una función estética: por ejemplo, no siempre son los argumentos de contenido los que explican la preferencia de los infantes por los contenidos mediáticos. Las historias que se ofrecen con los juegos de video o las caricaturas - entre otros- muestran cómo están presentes también ciertos efectos sonoros, de velocidad, estímulos visuales — multi-modalidad (Kress, 2010) -, etc., que, desde el punto de vista mimético (Wulf y Gebauer, 1995), empiezan a hacer parte también de nuestra velocidad de reacción, de nuestra gestualidad y de nuestras actitudes y hábitos corporales.

En la medida en que cada vez más se expande el internet, también las formas de estructuración de los grupos se reconfiguran. Ello les exige a los niños y las niñas 
trascender ciertos límites - locales, familiares, su entorno cercano- - y asumir otros modos de ver, pensar, sentir y experienciar como formas de vida propia. En lo que tiene que ver con el comportamiento social — consumista (Buckingham, 2013) —, los medios permiten orientaciones, por ejemplo, con respecto a la moda, a los estilos de vida, a ciertas jergas y, en general, a ciertos tipos de trato entre sí por parte de niños y niñas. Los medios también ayudan entonces a satisfacer diferentes necesidades de los infantes. Especialmente mediante la publicidad se promueven ciertas necesidades que bien aprovecha el mercado para sus propósitos. Un punto bastante problemático en este sentido es cuando con las ofertas comerciales publicitarias no se diferencia entre la ficción y el contenido, y con ello se afecta de diferentes maneras a los infantes. Diferencias que muchas veces ellos no están en condiciones de procesar y elaborar (ficción y realidad), pues, la mayoría de las veces, no se confrontan con los media y sus ofertas de un modo cuestionador y reflexivo. Entonces, no son solo los gustos, los conocimientos, las cuestiones valorativas y afectivas, sino también las formas de interactuar y de establecer y adscribirse a ciertos grupos las cosas que se aprenden desde muy temprano gracias a la ayuda de los diferentes medios. Éstos son un agente socializador.

\section{A modo de conclusión}

Así, como en las actuales teorías de la socialización, cuando se habla de socialización mediática se cuestiona la idea de un efecto unidireccional y se plantea, más bien, que se trata de un proceso interactivo en el que los individuos - los infantes- cumplen un papel activo. Las reflexiones sobre la socialización mediática abarcan los aspectos y efectos tanto negativos como positivos de los media (Vollbrecht, 2003, p. 13). La socialización mediática se puede ver como el aprendizaje no planeado - pero con reglas, e intencionalidades - de actitudes, formas de comportamiento mediante cierto tipo de estímulos (Lukesch, 2008). Se puede entender la socialización mediática como el resultado de influjos socializadores dado por el uso de los medias, lo cual no solo abarca el uso como tal, sino también la transformación de las actividades, comportamientos, situaciones —o marcos en el sentido de Goffman (1977) —, rituales, comportamientos, interacciones, etc., gracias a su presencia (por ejemplo, cuando me levanto una de mis acciones rutinizadas es ubicar mi celular, así no lo vaya a usar en el sentido estricto. El artefacto transforma mis prácticas, mis rutinas de acción).

En ese sentido también el cuerpo y los artefactos son parte del orden social e, igualmente, se encuentran involucrados en los procesos de socialización. Se vuelve claro entonces que algunas prácticas se encuentran en una relación estrecha con los artefactos y que se originan a partir de ellos. Por ejemplo, las prácticas de escritura, que van desde el desarrollo de la escritura pasando por la imprenta hasta la escritura por computadores, se encuentran estrechamente ligadas a artefactos técnicos y tienen efectos en las prácticas de comunicación y transmisión del saber, en las formas de archivar la información y también en la interacción, pues, por ejemplo, un encuentro entre amigos ya hoy puede tener lugar por medio de la escritura a través de chats. 
Mediante los medios se inician procesos de cambio de los individuos. Esto tiene que ver con procesos cotidianos de organización del mundo, lo que lleva a preguntarse por cómo los individuos consiguen en las situaciones cotidianas adscribirles sentido y a lo que allí sucede o hace presencia (circunstancias), y lleva a que se pregunte también por cómo se llega a concordancias sociales a partir de esas adscripciones de sentido. Podemos decir con Goffman (1977), que el individuo vive su mundo como una corriente de situaciones (no el hombre y sus circunstancias, sino las circunstancias y sus hombres). Y para otorgarles sentido a esas situaciones aplica o utiliza uno o varios marcos (frames) que le dan una explicación de lo que allí sucede y de cómo se puede definir a partir de allí un comportamiento adecuado. Esos marcos o situaciones -en los que están presentes cada vez más los media - se basan, en su capa más profunda, en normativas e instituciones sociales.

El concepto de socialización mediática comprende entonces tanto las características centrales de la socialización como también las de los medios, como su carácter educativo-formativo, sus efectos, influjos, identificaciones, consumos y símbolo de estatus. Sus consecuencias marcan a los infantes en sus procesos de socialización individual en relación con su familia, con la escuela y con los grupos de pares. Así, la reflexión sobre la socialización mediática se ocupa del papel que cumplen los media en los procesos de desarrollo social de los individuos. Los medios se comportan como agentes socializadores y son parte del entorno social y material con el que se confrontan de manera activa los infantes — también jóvenes y adultos-.

\section{Referencias}

Baacke, D (1998). Die 6-12 Jährigen. Einführung in die Probleme des Kinderalters. Weinehim y Basel: Beltz Verlag.

Brigido, A. (2006). Sociología de la educación. Temas y perspectivas fundamentales. Córdoba, Argentina: Ed. Brujas.

Bronfenbrenner, U. (1979). La ecología del desarrollo humano. Experimentos en entornos naturales y diseñados. Barcelona: Paidós.

Buckingham, D. (2002). Crecer en la era de los medios electrónicos: tras la muerte de la infancia. Madrid: Morata.

Buckingham, D. (2013). La infancia materialista. Crecer en la cultura consumista. Madrid: Morata.

Dubet, F. y Martuccelli, D. (1996). Teorías de la socialización: una definición sociológica. Revista francesa de Sociología, 37, 511-535.

Durkheim, E. (1976). La educación como socialización. Salamanca: Sígueme.

Gebauer, G. y Wulf, Ch. (1995). Mimesis. Berkeley/Los Angeles, University of California Press. (Ed. alem. 1992).

Goffman, E. (1977). Rahmen-Analyse. Ein Versuch über die Organisation von Alltagserfahrungen. Frankfurt am Main: suhrkamp Verlag. 
Graviz, A. (2010). Pedagogía mediática - aprendizaje e interculturalidad. Revista de Educación, 1(1), 97-110.

Grundmann, M. \& Kunze, I. (2008). Systematische Sozialraumforschung: Urie Bronfenbrenners Ökologie der menschlichen Entwicklung und die Modellierung mikrosozialer Raumgestaltung. En: Kessel, F. y Reutlinger, Chr. (Eds.), Schlüsselwerke der Sozialraumforschung. Wiesbaden: VS Verlag für Sozialwissenschaften.

Hurrelmann, K. (2006). Einfuihrung in die Sozialisationstheorie. Weinheim: Beltz Verlag.

Kleemann, F., Krähnke, U. \& Matuschek, I. (2013). Interpretative Sozialfoschung. Eine Einführung in die Praxis des Interpretierens. Wiesbaden: Springer VS.

Kress, G. (2009). Multimodality. A social semiotic approach to contemporary communication. New York: Routlege.

Kübler, H.-D. (2009). Mediensozialisation - ein Desiderat zur Erforschung von Medienwelten: Versuch einer Standortbestimmung und Perspektivik. Diskurs Kindheits- und Jugendforschung, 1(4), 7-26. Recuperado de http://nbn-resolving. de/urn:nbn:de:0168-ssoar-334439.

Lukesch, H. (2008). Sozialisation durch Massenmedien. En K. Hurrelmann, M. Grundmann \& S. Walper (Eds.), Handbuch Sozialisationsforschung. Wiesbaden: VS Verlag für Sozialwissenschaften.

Parsons, T. \& Bales, R. F. y otros (1956). Family, socialization and interaction process. London: Routledge \& Kegan Paul.

Parsons, T. (1999). El sistema social. Madrid: Alianza editorial.

Racioppe, B. (2008). Jóvenes y TICs. Modos de socialización y construcción de identidad. Ponencia. $10^{\circ}$ Congreso REDCOM "Conectados, Hipersegmentados y Desinformados en la Era de la globalización. Universidad Católica de Salta.

Schorb, B. (2005). Sozialisation. En J. Hüther \& B. Schorb, Grundbegriffe Medienpädagogik. München: KoPäd Verlag.

Schorb, B., Mohn, E. \& Theunert, H. (1991). Sozialisation durch (Massen-) Medien. En K. Hurrelmann \& D. Ulich (Eds.), Neues Handbuch der Sozialisationsforschung. Weinheim y Basel: Beltz Verlag.

Süss, D. (2004). Mediensozialisation von Heranwachsenden. Dimension - Konstanten - Wandel. Wiesbaden: VS Verlag für Sozialwissenschaften.

Theunert, H., Lenssen, M. \& Schorb, B. (1995). "Wir gucken besser fern als ihr!" Fernsehen für Kinder. München: KoPäd Verlag.

Vollbrecht, R. (2003). Aufwachsen in Medienwelten. En: K. Fritz, S. Sting \& R. Vollbrecht (Eds.), Mediensozialisation - Pädagogische Perspektiven des Aufwachsens in Medienwelten. Opladen: Leske + Budrich.

Zimmermann, P. (2006). Grundwissen Sozialisation. Einführung zur Sozialisation Kindes- und Jugendalter. Wiesbaden: VS Verlag für Sozialwissenschaften. 